\title{
UNSCENTED KALMAN FILTER FOR SPACECRAFT ATTITUDE ESTIMATION USING QUATERNIONS AND EULER ANGLES
}

\author{
R. V. Garcia (1), H. K. Kuga (2), M. C. Zanardi (3) \\ (1)(2) Space Mechanics and Control Division, National Institute for Space Research (INPE) São \\ Paulo, São José dos Campos 12227-010, Brazil, phone:55(12)3208-6199, \\ rovgarcia@yahoo.com.br,hkk@dem.inpe.br \\ (3) Department of Mathematics, Group of Orbital Dynamics and Planetology, São Paulo \\ University (UNESP), São Paulo, Guaratinguetá 12516-410, Brazil, phone: 55(12)3123-2830, \\ cecilia@feg.unesp.br
}

\begin{abstract}
A spacecraft attitude estimation approach based on the Unscented Kalman Filter is derived. For nonlinear systems the Unscented Kalman Filter uses a carefully selected set of sample points to map more accurately the probability distribution than the linearization of the standard Extended Kalman Filter, leading to faster convergence from inaccurate initial conditions in attitude estimation problems. The filter formulation is based on standard attitude-vector measurements using a gyro-based model for attitude propagation. This paper compares the performance of a new technique, the Unscented Kalman Filter, when two different mathematical constructs are used to represent the attitude: the Euler angles and quaternions. In this study, the attitude of satellite is estimated with real time algorithms using real data supplied by gyros, Earth sensors and Sun sensors that are on board of the CBERS-2 (China Brazil Earth Resources Satellite).
\end{abstract}

Keywords: Unscented Kalman Filter, real data, attitude estimation, quaternions, Euler angles.

\section{Introduction}

The attitude of a spacecraft is defined by its orientation in space related to some reference systems. The importance of determining the attitude is related not only to the performance of the attitude control system but also to the precise usage of information obtained by payload experiments performed by the satellite, e.g. [1].

The attitude estimation is the process of calculating the orientation of the spacecraft in relation to a reference system from data supplied by attitude sensors. Chosen the vectors of reference, an attitude sensor measures the orientation of these vectors with respect to the satellite reference system. Once these one or more vectors measurements are known, it is possible to compute the orientation of the satellite processing these vectors, using methods of attitude estimation. Several parameterizations can be used to represent the satellite attitude [2]. The most common way to represent the attitude of a spacecraft is a set of three Euler angles. These are popular because they are easy to understand and use. The main disadvantages of Euler angles are: (1) certain important functions of Euler angles have singularities, (2) they are less accurate than the unit quaternion, when used to integrate incremental changes in attitude over time, and (3) they use too much trigonometric functions, expensive for on board computers. These deficiencies in the Euler angles representation have led researchers to use unit quaternions as a parametrization of the attitude of a rigid body. The relevant functions of unit quaternions have no singularities and the representation is well-suited to integrate the angular velocity of a body over time. The main disadvantages of using unit quaternions are: (1) that the four quaternion parameters do not have a intuitive physical meanings, and (2) that a quaternion must have unity norm to be a pure rotation.

There are several methods for determining the attitude of a satellite. Each method is appropriate to a particular type of application and meets the needs such as: available time for processing and accuracy to be attained. However, all methods need observations that are obtained by means of 
sensors installed on the satellite. The sensors are essential for attitude estimation, because they measure its orientation relative to some referential, e.g. the Earth, the sun or a star. The method to estimate the attitude used is the Unscented Kalman Filter [3]. This method is capable of performing state estimation in nonlinear systems, besides taking into account measurements provided by different attitude sensors. This work considers real data supplied by gyroscopes, infrared Earth sensors and digital sun sensors. These sensors are on board of the CBERS-2 satellite (China-Brazil Earth Resources Satellite) [4], and the measurements were downlinked to the Satellite Control Centre of INPE (Brazilian Institute for Space Research).

\section{The Unscented Kalman Filter for attitude determination}

The inherent flaws of the Extended Kalman Filter (EKF) are due to its linearization approach [5] for calculating the mean and covariance of a random variable which undergoes a nonlinear transformation. The Unscented Kalman Filter (UKF) addresses these flaws by utilizing a deterministic "sampling" approach to calculate mean and covariance terms. Essentially, $2 n+1$, sigma points ( $n$ is the state dimension), are carefully chosen based on a square-root decomposition of the prior covariance [6]. These sigma points are propagated through the true nonlinearity, without approximation, and then a weighted mean and covariance is taken. The UKF has several advantages over the EKF, including: 1) the expected error is lower than the EKF, 2) the new filter can be applied to non-differentiable functions, 3) the new filter avoids the derivation of Jacobian matrices, and 4) the new filter is valid to higher-order expansions than the standard EKF.

Consider the nonlinear system model given by:

$\dot{\boldsymbol{x}}_{k}=f\left(\boldsymbol{x}_{k}, k\right)+G_{k} \boldsymbol{w}_{k}$

$\boldsymbol{y}_{k}=h\left(\boldsymbol{x}_{k}, k\right)+\boldsymbol{v}_{k}$

where $\boldsymbol{x}_{\mathrm{k}}$ is the $n \times 1$ state vector and $\boldsymbol{y}_{\mathrm{k}}$ is the $m \times 1$ measurement vector. The function $f$ is a possible nonlinear function of the state $\boldsymbol{x}_{\mathrm{k}} \in \mathfrak{R}^{\mathrm{n}}$ and the observation $\boldsymbol{y}_{\mathrm{k}} \in \mathfrak{R}^{\mathrm{m}}$ is often a nonlinear mapping of the current state. Both the dynamic model and the measurement model are inaccurate, due to modeling and/or sensor errors. This is described by the stochastic processes where we assume that the process noise $\boldsymbol{w}_{\mathrm{k}}$ and measurement-error noise $\boldsymbol{v}_{\mathrm{k}}$ are zero-mean Gaussian noise process with covariances given by $Q_{\mathrm{k}}$ and $R_{\mathrm{k}}$, respectively.

Given the state vector and the covariance matrix at step $k$-1, we compute a collection of sigma points, stored in the columns of the $n \times(2 n+1)$ sigma point matrix $\chi_{\mathrm{k}-1}$ where $n$ is the dimension of the state vector. The columns of $\chi_{\mathrm{k}-1}$ are computed by:

$$
\begin{aligned}
& \left(\boldsymbol{\chi}_{\mathrm{k}-1}\right)_{0}=\hat{\boldsymbol{x}}_{\mathrm{k}-1} \\
& \left(\boldsymbol{\chi}_{\mathrm{k}-1}\right)_{\mathrm{i}}=\hat{\boldsymbol{x}}_{\mathrm{k}-1}+\left(\sqrt{(n+\lambda) \mathrm{P}_{\mathrm{k}-1}}\right)_{\mathrm{i}} \quad i=1, \ldots, n \\
& \left(\boldsymbol{\chi}_{\mathrm{k}-1}\right)_{\mathrm{i}}=\hat{\boldsymbol{x}}_{\mathrm{k}-1}-\left(\sqrt{(n+\lambda) \mathrm{P}_{\mathrm{k}-1}}\right)_{\mathrm{i}-n} \quad i=n+1, \ldots 2 n
\end{aligned}
$$

in which $\lambda \in \Re,\left(\sqrt{(n+\lambda) \mathrm{P}_{\mathrm{k}-1}}\right)_{\mathrm{i}}$ is the ith column of the matrix square root of $(n+\lambda) \mathrm{P}_{\mathrm{k}-1}$ and the scalar $\lambda$ is a convenient parameter for exploiting knowledge about the higher moments of the given distribution. Note that we assume matrix $\left(\sqrt{(n+\lambda) \mathrm{P}_{\mathrm{k}-1}}\right)_{\mathrm{i}}$ as symmetric and positive definite which allows us to find the square root using a Cholesky decomposition. 
Once $\boldsymbol{\chi}_{\mathrm{k}-1}$ computed, the sigma points are propagated through the nonlinear system

$\left(\chi_{\mathrm{k}}\right)_{i}=f\left(\left(\chi_{\mathrm{k}-1}\right)_{i}\right), \quad i=0, \ldots 2 n$,

The posterior mean, $\hat{\boldsymbol{x}}_{\mathrm{k}}^{-}$, and covariance, $\mathrm{P}_{\mathrm{k}}^{-}$, are determined from the statistics of the propagated sigma points as follows

$\hat{\boldsymbol{x}}_{\mathrm{k}}^{-}=\sum_{i=0}^{2 n} W_{i}\left(\boldsymbol{\chi}_{\mathrm{k}}\right)_{i}$

$\mathrm{P}_{\mathrm{k}}^{-}=\sum_{i=0}^{2 \mathrm{n}} W_{i}\left[\left(\boldsymbol{\chi}_{\mathrm{k}}\right)_{i}-\hat{\mathbf{x}}_{\mathrm{k}}^{-}\right]\left[\left(\boldsymbol{\chi}_{\mathrm{k}}\right)_{i}-\hat{\mathbf{x}}_{\mathrm{k}}^{-}\right]^{\mathrm{T}}+Q_{\mathrm{k}}$

where the weights are defined by: $W_{0}=\frac{\lambda}{(n+\lambda)}, W_{i}=\frac{1}{2(n+\lambda)}, i=1, \ldots ., 2 n$.

To compute the correction step, first we must transform the columns of $\boldsymbol{\chi}_{\mathrm{k}}$ through the measurement function to $\mathbf{Y}_{\mathrm{k}}$. In this way

$$
\begin{aligned}
& \left(\mathbf{Y}_{\mathrm{k}}\right)_{i}=h\left(\left(\boldsymbol{\chi}_{\mathrm{k}}\right)_{i}\right), \quad i=0, \ldots 2 n \\
& \hat{\mathbf{y}}_{\mathrm{k}}^{-}=\sum_{i=0}^{2 \mathrm{n}} W_{i}\left(\mathbf{Y}_{\mathrm{k}}\right)_{i}
\end{aligned}
$$

With the mean measurement vector, $\hat{\mathbf{y}}_{\mathrm{k}}^{-}$, we compute the a posteriori state estimate using

$$
\hat{\boldsymbol{x}}_{k}=\hat{\boldsymbol{x}}_{k}^{-}+K_{k}\left(\boldsymbol{y}_{k}-\hat{\boldsymbol{y}}_{k}^{-}\right)
$$

where $K_{\mathrm{k}}$ is the Kalman gain. In the UKF formulation, the Kalman gain is defined by

$$
K_{k}=P_{x y} P_{y y}^{-1}
$$

with

$$
\begin{aligned}
& P_{x y}=\sum_{i=0}^{2 n} W_{i}\left[\left(\boldsymbol{\chi}_{k}\right)_{i}-\hat{\boldsymbol{x}}_{k}^{-}\right]\left[\left(\boldsymbol{Y}_{k}\right)_{i}-\hat{\boldsymbol{y}}_{k}^{-}\right]^{T} \\
& P_{y y}=\sum_{i=0}^{2 n} W_{i}\left[\left(\boldsymbol{Y}_{k}\right)_{i}-\hat{\boldsymbol{y}}_{k}^{-}\right]\left[\left(\boldsymbol{Y}_{k}\right)_{i}-\hat{\boldsymbol{y}}_{k}^{-}\right]^{T}+R
\end{aligned}
$$

Finally, the last calculation in the correction step is to compute the a posterior estimate of the error covariance given by

$$
\mathrm{P}_{\mathrm{k}}=\mathrm{P}_{\mathrm{k}}^{-}-K_{\mathrm{k}} \mathrm{P}_{\mathrm{yy}} K_{\mathrm{k}}^{\mathrm{T}}
$$




\section{Representation of Artificial Satellite Attitude}

There are different types of parameters to represent the attitude of a satellite [7], with highlights to the Euler angles representation and representation by quaternions.

\subsection{Attitude by Euler Angles}

The attitude of an artificial satellite is directly related to its orientation in space. Through the attitude one can know the spatial orientation of the satellite, since in most cases it can be considered as a rigid body, where the attitude is expressed by the relationship between two coordinate systems, one fixed on the satellite and another associated with a reference system, e.g. inertial system. For a good performance of the mission it is essential that the satellite be stabilized in relation to a specified attitude. The attitude stabilization is done by the on board attitude control, which is designed to acquire and maintain the satellite in a pre-defined attitude. The CBERS-2 attitude is stabilized in three axes, geopointed, and can be described with respect to the orbital system. In this reference system, the movement around the direction of the orbital velocity is called roll $\phi$. The movement around the direction normal to the orbit is called pitch $\theta$, and finally the movement around the direction Nadir/Zenith is called yaw $\psi$.

To transform a vector represented in a given reference into another it is necessary to define a matrix of direction cosines (T), where its elements are written in terms of Euler angles $(\phi, \theta, \psi)$. The rotation sequence used in this work for the Euler angles was the 3-2-1, where the coordinate system fixed in the body of the satellite $(x, y, z)$ is related to the orbital coordinate system $(x o, y o, z o)$ through the following sequence of rotations [4]:

- $1^{\text {st }}$ rotation of an angle $\psi$ (yaw angle) around the zo axis;

$-2^{\text {nd }}$ rotation of an angle $\theta$ (pitch angle) around an intermediate axis $y^{\prime}$;

$-3^{\text {rd }}$ rotation of an angle $\phi$ (roll angle) around the $x$ axis.

The matrix obtained through the 3-2-1 rotation sequence is given by:

$$
\mathrm{T}=\left[\begin{array}{ccc}
\mathrm{C} \theta \mathrm{C} \psi & \mathrm{C} \theta \mathrm{S} \psi & -\mathrm{S} \theta \\
\mathrm{S} \phi \mathrm{S} \theta \mathrm{C} \psi-\mathrm{C} \phi \mathrm{S} \psi & \mathrm{S} \phi \mathrm{S} \theta \mathrm{S} \psi+\mathrm{C} \phi \mathrm{C} \psi & \mathrm{S} \phi \mathrm{C} \theta \\
\mathrm{C} \phi \mathrm{S} \theta \mathrm{C} \psi+\mathrm{S} \phi \mathrm{S} \psi & \mathrm{C} \phi \mathrm{S} \theta \mathrm{S} \psi-\mathrm{S} \phi C \psi & \mathrm{C} \theta \mathrm{C} \phi
\end{array}\right]
$$

where $\mathrm{T}_{\mathrm{bo}}$ is the matrix of direction cosines with $\mathrm{S}=\sin , \mathrm{C}=\cos$.

By representing the attitude of a satellite with Euler angles, the set of kinematic equations are given by $[4,8]$ :

$$
\left[\begin{array}{c}
\dot{\phi} \\
\dot{\theta} \\
\dot{\psi}
\end{array}\right]=\left[\begin{array}{ccc}
1 & \mathrm{~S} \phi \operatorname{Tan} \theta & \mathrm{C} \phi \operatorname{Tan} \theta \\
0 & \mathrm{C} \phi & -\mathrm{S} \phi \\
0 & \mathrm{~S} \phi / C \theta & \mathrm{C} \phi / C \theta
\end{array}\right]\left[\begin{array}{c}
\hat{\omega}_{\mathrm{x}} \\
\hat{\omega}_{\mathrm{y}} \\
\hat{\omega}_{\mathrm{z}}
\end{array}\right]-\mathrm{T}\left[\begin{array}{c}
0 \\
-\omega_{o} \\
0
\end{array}\right]
$$

where $\omega_{0}$ is the orbital angular velocity and $\hat{\omega}_{\mathrm{x}}, \hat{\omega}_{\mathrm{y}}, \hat{\omega}_{\mathrm{z}}$ are the components of the angular velocity on the satellite system. 
Performing the necessary simplifications (small Euler angles) in Eq. 13, the differential equations of attitude are modeled by:

$$
\begin{aligned}
& \dot{\phi}(\mathrm{t})=\omega_{0} \sin \hat{\psi}+\hat{\omega}_{\mathrm{x}}+\hat{\theta} \hat{\omega}_{\mathrm{z}} \\
& \dot{\theta}(\mathrm{t})=\omega_{0} \cos \hat{\psi}+\hat{\omega}_{\mathrm{y}}+\hat{\phi} \hat{\omega}_{\mathrm{z}} \\
& \dot{\psi}(\mathrm{t})=\omega_{0}(\hat{\theta} \sin \hat{\psi}-\hat{\varphi} \cos \hat{\psi})+\hat{\omega}_{\mathrm{z}}+\hat{\phi} \hat{\omega}_{\mathrm{y}}
\end{aligned}
$$

The advantage of attitude representation by Euler angles is to use only three parameters. However, this representation has the disadvantage of the possible occurrence of singularities in the mathematical modeling of the satellite's motion, besides the need of trigonometric function computations.

\subsection{Attitude by Quaternions}

The quaternions are useful in inertial navigation systems on board the satellite, shows no singularities in the kinematic equations, gives a rule of algebraic products suitable for successive rotations, and the rotation matrix in terms of the quaternion does not depend on trigonometric functions. However, the quaternions have one redundant component (they are 4) with reference to Euler angles (they are 3 ) and does not have an immediate physical interpretation.

The quaternion set is defined by

$\mathrm{q} \equiv\left[\begin{array}{ll}\mathbf{q}^{\mathrm{T}} & \mathrm{q}_{4}\end{array}\right]^{\mathrm{T}}$,

with $\mathbf{q} \equiv\left[\begin{array}{lll}q_{1} & q_{2} & q_{3}\end{array}\right]^{T}=\hat{\mathbf{e}} \sin (\vartheta / 2)$ and $q_{4}=\cos (\vartheta / 2)$,

where $\hat{\mathbf{e}}$ is the axis of rotation and $\vartheta$ is the angle of rotation. Since a four-dimensional vector is used to describe three dimensions, the quaternion components cannot be independent from each other. The quaternion satisfies a single constraint given by $\mathrm{q}^{\mathrm{T}} \mathrm{q}=1$. The attitude matrix is related to the quaternion by [9]:

$$
\mathrm{T}(\mathrm{q})=\left[\begin{array}{ccc}
\mathrm{q}_{1}^{2}-\mathrm{q}_{2}^{2}-\mathrm{q}_{3}^{2}+\mathrm{q}_{4}^{2} & 2\left(\mathrm{q}_{1} \mathrm{q}_{2}+\mathrm{q}_{3} \mathrm{q}_{4}\right) & 2\left(\mathrm{q}_{1} \mathrm{q}_{3}-\mathrm{q}_{2} \mathrm{q}_{4}\right) \\
2\left(\mathrm{q}_{1} \mathrm{q}_{2}-\mathrm{q}_{3} \mathrm{q}_{4}\right) & -\mathrm{q}_{1}^{2}+\mathrm{q}_{2}^{2}-\mathrm{q}_{3}^{2}+\mathrm{q}_{4}^{2} & 2\left(\mathrm{q}_{2} \mathrm{q}_{3}+\mathrm{q}_{1} \mathrm{q}_{4}\right) \\
2\left(\mathrm{q}_{1} \mathrm{q}_{3}+\mathrm{q}_{2} \mathrm{q}_{4}\right) & 2\left(\mathrm{q}_{2} \mathrm{q}_{3}-\mathrm{q}_{1} \mathrm{q}_{4}\right) & -\mathrm{q}_{1}^{2}-\mathrm{q}_{2}^{2}+\mathrm{q}_{3}^{2}+\mathrm{q}_{4}^{2}
\end{array}\right]
$$

The quaternion kinematics equation is given by:

$$
\dot{\mathrm{q}}(\mathrm{t})=\frac{1}{2} \Omega(\hat{\omega}) \mathrm{q}(\mathrm{t})
$$

with: $\Omega(\boldsymbol{\omega})=\left(\begin{array}{cccc}0 & \omega_{\mathrm{z}} & -\omega_{\mathrm{y}} & \omega_{\mathrm{x}} \\ -\omega_{\mathrm{z}} & 0 & \omega_{\mathrm{x}} & \omega_{\mathrm{y}} \\ \omega_{\mathrm{y}} & -\omega_{\mathrm{x}} & 0 & \omega_{\mathrm{z}} \\ -\omega_{\mathrm{x}} & -\omega_{\mathrm{y}} & -\omega_{\mathrm{z}} & 0\end{array}\right)$ 
A major advantage of using the quaternion is that the kinematics equation is linear in the quaternion and is also free of singularities. In this paper, it is assumed that the gyro data are assembled in a fixed rate and that the angular spin velocity vector in a satellite system, $\omega$, is constant over the interval of sampling. Then a solution of Eq. 16 is [7, 8]:

$$
\mathrm{q}\left(\mathrm{t}_{\mathrm{k}}\right)=\Phi_{\mathrm{q}}(\Delta \mathrm{t}, \boldsymbol{\omega}) \mathrm{q}\left(\mathrm{t}_{\mathrm{k}-1}\right)
$$

where $\Delta \mathrm{t}$ is the sampling interval; $\mathrm{q}\left(\mathrm{t}_{\mathrm{k}-1}\right)$ is the quaternion at time $\mathrm{t}_{\mathrm{k}-1} ; \mathrm{q}\left(\mathrm{t}_{\mathrm{k}}\right)$ is the propagated quaternion to time $t_{k}$; e $\Phi_{\mathrm{q}}$ is the transition matrix that computes the system from time $t_{k-1}$ to $t_{k}$, given by:

$$
\Phi_{\mathrm{q}}(\Delta \mathrm{t}, \vec{\omega})=\operatorname{ICos}\left(\frac{|\boldsymbol{\omega}| \Delta \mathrm{t}}{2}\right)+\frac{1}{|\boldsymbol{\omega}|} \operatorname{Sin}\left(\frac{|\boldsymbol{\omega}| \Delta \mathrm{t}}{2}\right) \Omega(\boldsymbol{\omega})
$$

If the bias term is included in the formulation of the quaternion propagation, i.e., $\hat{\boldsymbol{\omega}}=\boldsymbol{\omega}-\boldsymbol{\varepsilon}$, where $\boldsymbol{\varepsilon}$ is a vector of gyro biases, then a system state composed by quaternions and gyro biases will have the following transition matrix $[3,5]$ :

$\Phi=\left[\begin{array}{cc}\Phi_{\mathrm{q}} & \Psi \\ 0_{3 \times 4} & \mathrm{I}_{3 \times 3}\end{array}\right]$

where $\Psi=-\frac{1}{2} \int_{t_{\mathrm{o}}}^{\mathrm{t}} \Phi_{\mathrm{q}} \Xi \mathrm{dt}$ and the $\Xi$ matrix is given by:

$$
\Xi=\left[\begin{array}{ccc}
q_{4} & -q_{3} & q_{2} \\
q_{3} & q_{4} & -q_{1} \\
-q_{2} & q_{1} & q_{4} \\
-q_{1} & -q_{2} & -q_{3}
\end{array}\right]
$$

Note that the system is still linear with respect to system state composed by quaternions and biases. Therefore the transition matrix also assures the needed coupling between quaternions and biases to be taken into account in the covariance computations.

With such considerations, the quaternion approach makes the system dynamics to be fully linear, i.e.

$$
\dot{\boldsymbol{x}}_{k}=\mathrm{F}_{\mathrm{k}} \boldsymbol{x}_{k}+\mathrm{G}_{k} \boldsymbol{w}_{k}
$$

Thus in the prediction of state and covariance cycle, the conventional linear Kalman filter can be used so that one saves processing cost. Only the measurement equations are still non linear, and the Unscented transformation should be used, with the corresponding sigma-point measurement update cycle being implemented in the UKF.

\section{The Measurements System of Satellite}

This section describes the mathematical models of the sensors used for attitude determination. The modeled sensors are: Gyros, Digital Sun sensor and Infrared Earth sensor. 


\subsection{The Model for Gyros}

The advantage of a gyro is that it can provide the angular displacement and/or angular velocity of the satellite directly. However, gyros have an error due to drifting, meaning that their measurement error increases with time. In this work, the rate-integration gyros (RIGs) are used to measure the angular velocities of the body axes of the satellite. The mathematical model of the RIGs is [8]:

$$
\Delta \boldsymbol{\Theta}_{\mathrm{i}}=\int_{0}^{\Delta \mathrm{t}}\left(\boldsymbol{\omega}_{\mathrm{i}}+\boldsymbol{\varepsilon}_{\mathrm{i}}\right) \mathrm{dt} \quad(\mathrm{i}=x, y, z)
$$

where $\Delta \Theta$ are the angular displacements of the satellite axes in a time interval $\Delta \mathrm{t}$, and $\varepsilon_{\mathrm{i}}$ are components of bias of the gyroscope.

Thus, the measured components of the angular velocity of the satellite are given by:

$$
\hat{\boldsymbol{\omega}}=\left(\frac{\Delta \Theta}{\Delta \mathrm{t}}\right)-\hat{\boldsymbol{\varepsilon}}-\eta=\mathbf{g}-\boldsymbol{\varepsilon}-\eta_{\mathrm{att}}
$$

where $\mathbf{g}(\mathrm{t})$ is the output vector of the gyroscope; and $\eta(t)$ represents a Gaussian white noise process covering all the remaining unmodelled effects:

$$
\mathrm{E}\left[\eta_{\mathrm{att}} \eta_{\mathrm{att}}^{\mathrm{T}}\right]=\sigma_{\mathrm{att}}^{2}
$$

\subsection{The Measurement Model for Infrared Earth Sensors (IRES)}

One way to compensate for the drifting errors present in gyros is to use the earth sensors. These sensors are located on the satellite and aligned with their axes of roll and pitch. In this paper, two earth sensors are used, with one measuring the roll angle and the other measuring the pitch angle. In principle, an earth sensor cannot measure the yaw angle.

The measurement equations for the earth sensors are given as:

$\phi_{\mathrm{H}}=\phi+\eta_{\phi_{\mathrm{H}}}$
$\theta_{\mathrm{H}}=\theta+\eta_{\theta_{\mathrm{H}}}$

where $\eta_{\phi_{\mathrm{H}}}$ and $\eta_{\theta_{\mathrm{H}}}$ are the white noise representing the small remaining misalignment, installation and/or assembly errors assumed gaussian:

$\mathrm{E}\left[\eta_{\phi_{\mathrm{H}}} \eta_{\phi_{\mathrm{H}}}^{\mathrm{T}}\right]=\mathrm{E}\left[\eta_{\theta_{\mathrm{H}}} \eta_{\theta_{\mathrm{H}}}^{\mathrm{T}}\right]=\sigma_{\mathrm{IRES}}^{2}$

\subsection{The Measurement for Digital Solar Sensors (DSS)}

Since an earth sensor is not able to measure the yaw angle, the digital solar sensors are used by the Attitude Control System in order to overcome this problem. However, these sensors do not provide direct measurements but coupled angle of pitch $\left(\alpha_{\theta}\right)$ and yaw $\left(\alpha_{\psi}\right)$. The measurement equations for the solar sensor are obtained as follows [4]: 


$$
\alpha_{\psi}=\tan ^{-1}\left(\frac{-S_{y}}{S_{x} \cos \left(60^{\circ}\right)+S_{z} \cos \left(150^{\circ}\right)}\right)+\eta_{\alpha_{\psi}}
$$

when $\left|S_{x} \cos \left(60^{\circ}\right)+S_{z} \cos \left(150^{\circ}\right)\right| \geq \cos \left(60^{\circ}\right)$, and

$$
\alpha_{\theta}=24^{\circ}-\tan ^{-1}\left(\frac{S_{x}}{S_{z}}\right)+\eta_{\alpha_{\theta}}
$$

when $\left|24^{\circ}-\tan ^{-1}\left(\frac{\mathrm{S}_{\mathrm{x}}}{\mathrm{S}_{\mathrm{z}}}\right)\right|<60^{\circ}$,

where $\eta_{\alpha_{\psi}}$ and $\eta_{\alpha_{\theta}}$ are the white noise representing the small remaining misalignment, installation and/or assembly errors assumed gaussian:

$$
\mathrm{E}\left[\eta_{\alpha_{\psi}} \eta_{\alpha_{\psi}}^{\mathrm{T}}\right]=\mathrm{E}\left[\eta_{\alpha_{\theta}} \eta_{\alpha_{\theta}}^{\mathrm{T}}\right]=\sigma_{\mathrm{DSS}}^{2}
$$

The conditions are such that the solar vector is in the field of view of the sensor, and $S_{x}, S_{y}, S_{z}$ are the components of the unit vector associated to the sun vector in the satellite system and given by:

$$
\begin{aligned}
& S_{x}=S_{0 x}-\hat{\psi} S_{0 y}-\hat{\theta} S_{0 z} \\
& S_{y}=S_{0 y}-\hat{\psi} S_{0 x}-\hat{\phi} S_{0 z} \\
& S_{z}=S_{0 z}-\hat{\phi} S_{0 y}-\hat{\theta} S_{0 z}
\end{aligned}
$$

where $\mathrm{S}_{0 \mathrm{x}}, \mathrm{S}_{0 \mathrm{y}}, \mathrm{S}_{0 \mathrm{z}}$ are the components of the sun vector in the orbital coordinate system and $\hat{\phi}, \hat{\theta}, \hat{\psi}$ are the Euler angles estimated attitude.

\section{Results and Discussions}

Here, the results and the analysis from the algorithms developed to estimate the attitude are presented. To validate and to analyze the performance of the estimators, real sensors data from the CBERS-2 satellite were used. The CBERS-2 satellite was launched on October 21st, 2003. The measurements are for the 21st of April 2006, available to the ground system at a sampling rate of about 8.56 seconds for around 10 minutes. Indeed the on-board ACS (Attitude Control System) has full access to the sensor measurements sampled at the rate of $4 \mathrm{~Hz}$ for gyros, $1 \mathrm{~Hz}$ for Earth sensors and $0.25 \mathrm{~Hz}$ for sun sensors. However, owing to limited downlinked TM (telemetry), the ground system can recover telemetries for the sensors at around 9 seconds sampling and only during the satellite fly over the tracking station. This means that the ground system does not have the whole set of measurements available to the on-board ACS [10].

The algorithms were implemented through MatLab software. To check the performance the UKF using the Euler angles and the quaternions, their results were compared considering the following set of initial conditions: 


\section{- Initial Attitude:}

$\operatorname{roll}(\phi)=\operatorname{pitch}(\theta)=y a w(\psi)=0 \mathrm{deg}$;

- Initial Bias of Gyros:

$\varepsilon_{\mathrm{x}}=5.56 \mathrm{deg} / \mathrm{hour} ; \varepsilon_{\mathrm{y}}=0.87 \mathrm{deg} / \mathrm{hour} ; \varepsilon_{\mathrm{z}}=6.12 \mathrm{deg} / \mathrm{hour}$;

- Initial Covariance (P):

$\sigma_{\phi, \theta, \psi}^{2}=(0.5 \mathrm{deg})^{2}$ - error related to the attitude with Euler angles;

$\sigma_{\text {quat } 1,2,3}^{2}=\left(2.5 \times 10^{-3}\right)^{2}$ - error related to the attitude with quaternions;

$\sigma_{\text {quat } 4}^{2}=\left(1.9 \times 10^{-5}\right)^{2}$ - error related to the attitude with the portion of the quaternion vetorial;

$\sigma_{\text {biasx }, \mathrm{y}, \mathrm{z}}^{2}=(1 \mathrm{deg} / \mathrm{hour})^{2}$ - error related to the drift of gyro;

- Observation error Covariance $(\mathbf{R})$ :

$\sigma_{\text {DSS }}^{2}=(0.3 \mathrm{deg})^{2}-$ sun sensor;

$\sigma_{\text {IRES }}^{2}=(0.03 \mathrm{deg})^{2}-$ earth sensor;

- Dynamic Noise Covariance (Q):

$\sigma_{\text {AttEuler }}^{2}(0.5 \mathrm{deg})^{2}$ - error related to the attitude with Euler angles;

$\sigma_{\text {Attquat }}^{2}=\left(10^{-3}\right)^{2}-$ error related to the attitude with quaternions;

$\sigma_{\text {Dgyrox,y }}^{2}=(1 \mathrm{deg} / \mathrm{hour})^{2}$ - error related to the drift of gyro in $\mathrm{x}, \mathrm{y}$ axes;

$\sigma_{\text {Dgyroz }}^{2}=(1 \mathrm{deg} / \text { hour })^{2}-$ error related to the drift of gyro in $\mathrm{z}$ axis;

The real measurements obtained by the attitude sensors (digital sun sensors, infrared Earth sensors and gyros) are shown below in Fig. 1.
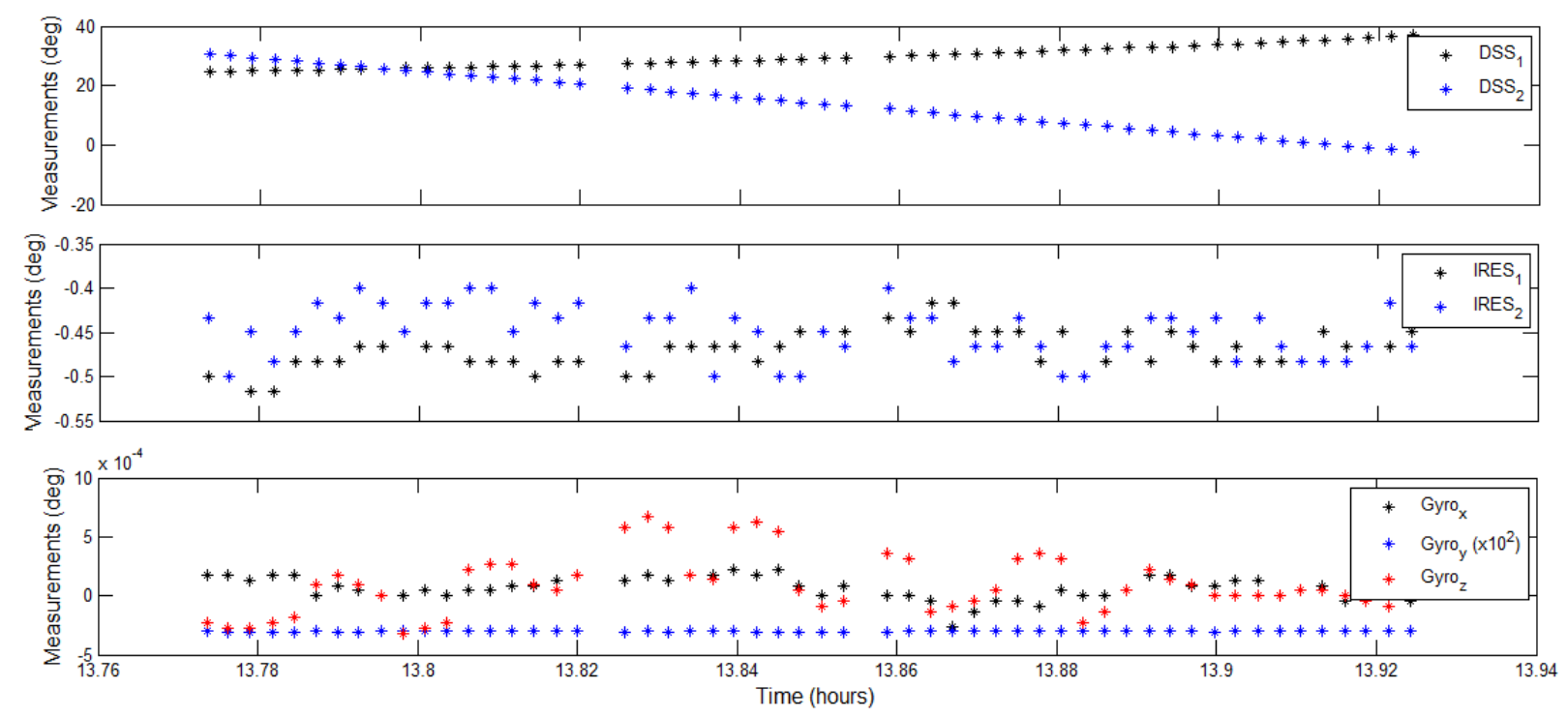

Figure 1. Real measurements supplied by attitude sensors

The next figures present the results obtained with the Unscented Kalman filter using the two different attitude parameterizations: Euler angles $\left(\mathrm{UKF}_{\mathrm{E}}\right)$ e quaternions $\left(\mathrm{UKF}_{\mathrm{Q}}\right)$.

In Fig. 2 and 3 it is observed the behavior of attitude and the biases of gyros during the period analyzed. The mean estimate to roll and pitch are in the order of $-0.47 \mathrm{deg}$ and $-0.46 \mathrm{deg}$, respectively, for both estimators $\left(\mathrm{UKF}_{\mathrm{E}}\right.$ and $\left.\mathrm{UKF}_{\mathrm{Q}}\right)$. For yaw a random shape is not observed and its mean value is around $-1.35 \mathrm{deg}$ for $\mathrm{UKF}_{\mathrm{Q}}$ and -1.48 deg for $\mathrm{UKF}_{\mathrm{E}}$. In Fig. 3 it is observed the bias estimates behavior, where the $\mathrm{UKF}_{\mathrm{Q}}$ still shows a small variation while the $\mathrm{UKF}_{\mathrm{E}}$ seems to be converged. 

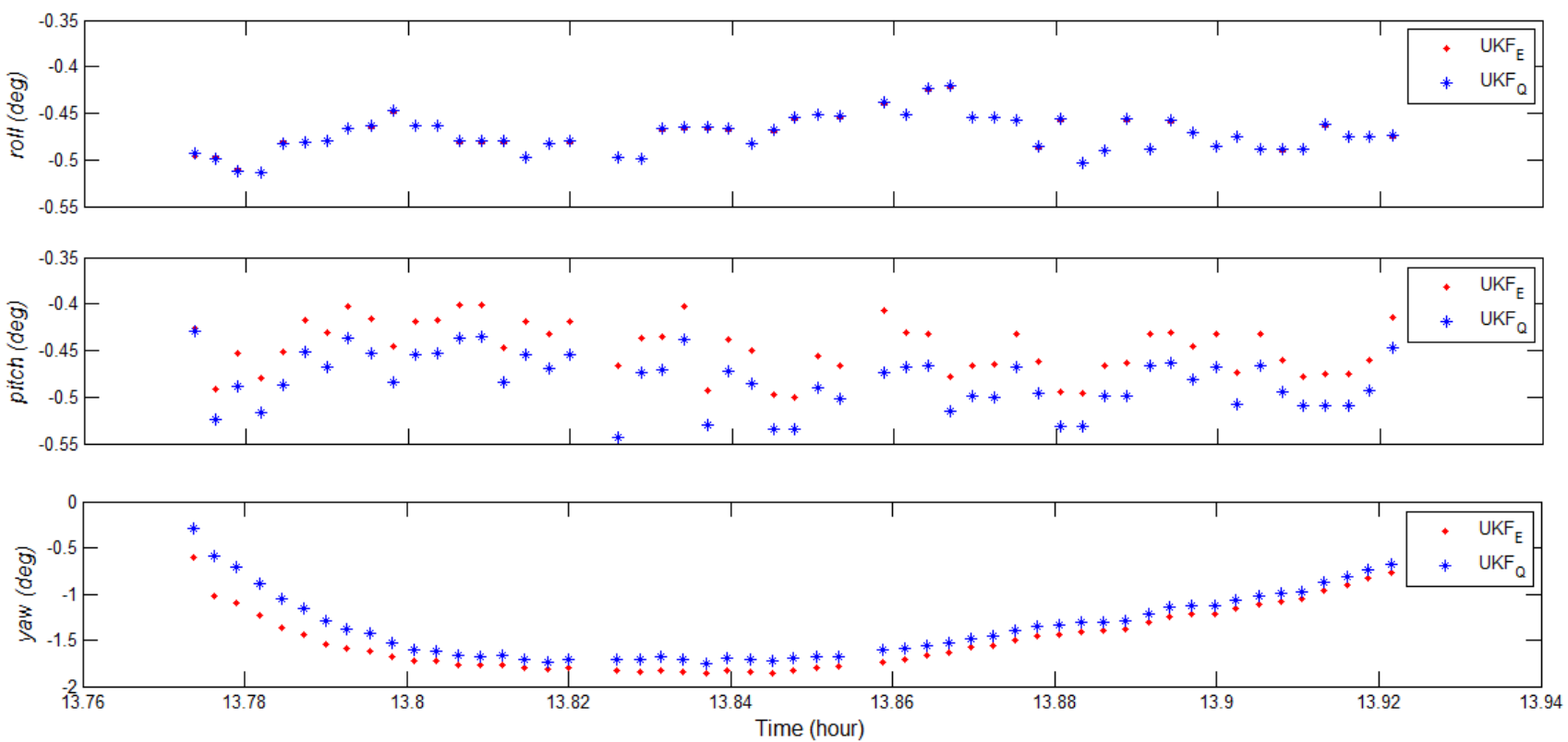

Figure 2. Estimated Attitude
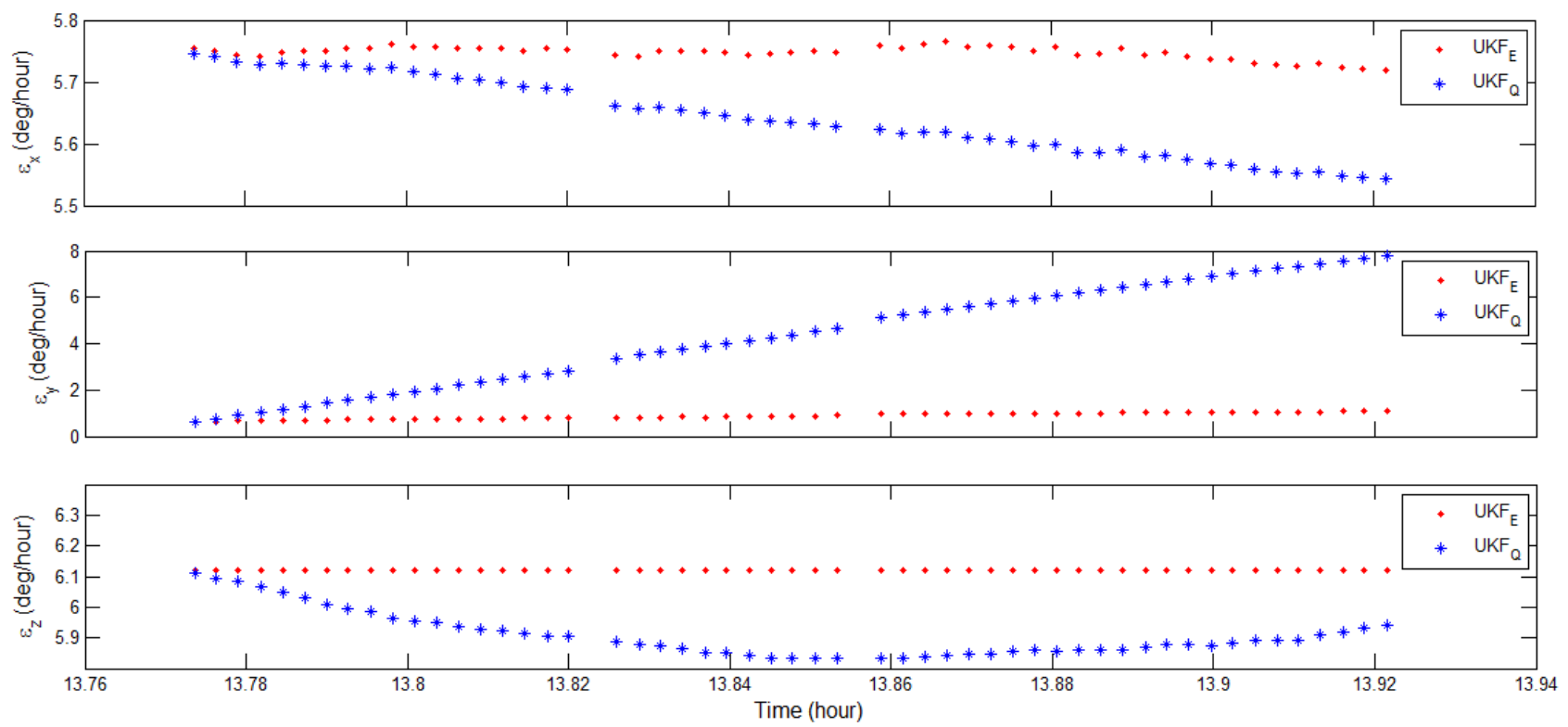

Figure 3. Estimated gyros biases

The Fig. 4 and 5 presents the standard deviations for both estimators for the attitude and the bias of the gyro. It is observed that the standard deviations for attitude and gyro bias decreases with a tendency to stabilize around a value for both estimators. However, it is noticed that $\mathrm{UKF}_{\mathrm{Q}}$ is more optimistic with its covariance going most of the times below the covariance of $\mathrm{UKF}_{\mathrm{E}}$. 

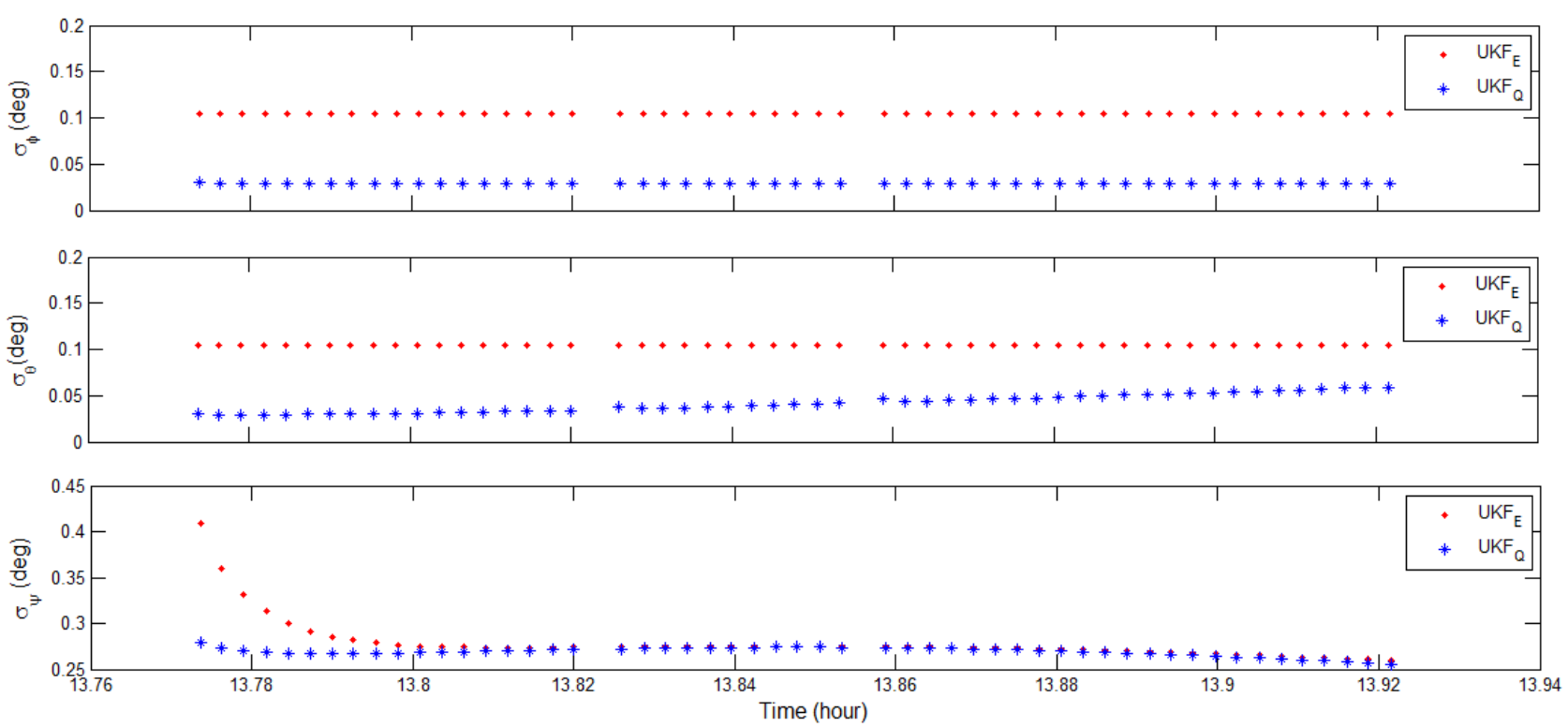

Figure 4. Attitude Error Estimation
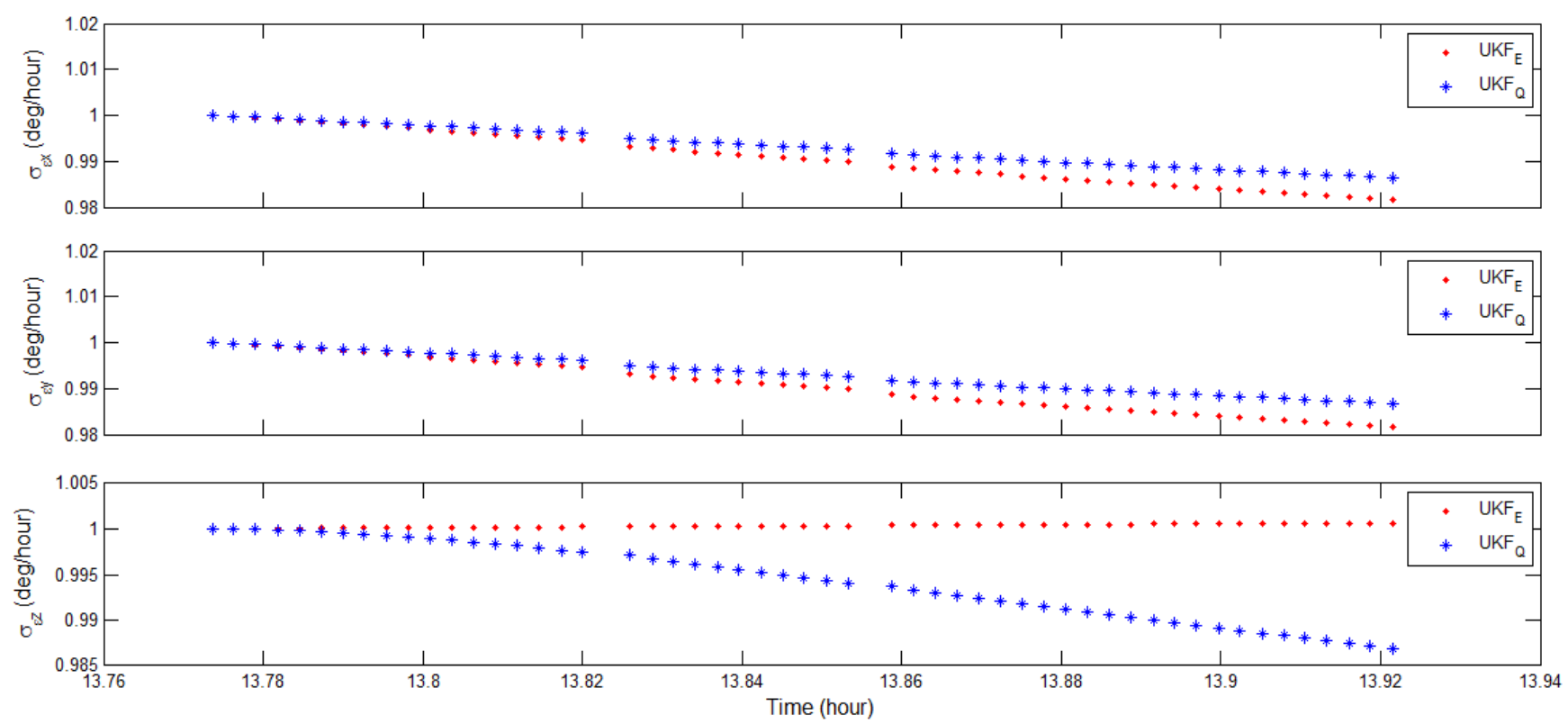

Figure 5. Biases Estimation Errors

In figures 6 and 7, we can see the residues of sun sensors and Earth sensors, respectively. Residuals of $\mathrm{UKF}_{\mathrm{E}}$ looks better in the sense of zero mean statistics. $\mathrm{UKF}_{\mathrm{Q}}$ residuals still present some trends which can mostly be assigned to the still not converged gyro bias estimates of the $\mathrm{UKF}_{\mathrm{Q}}$ algorithm. 

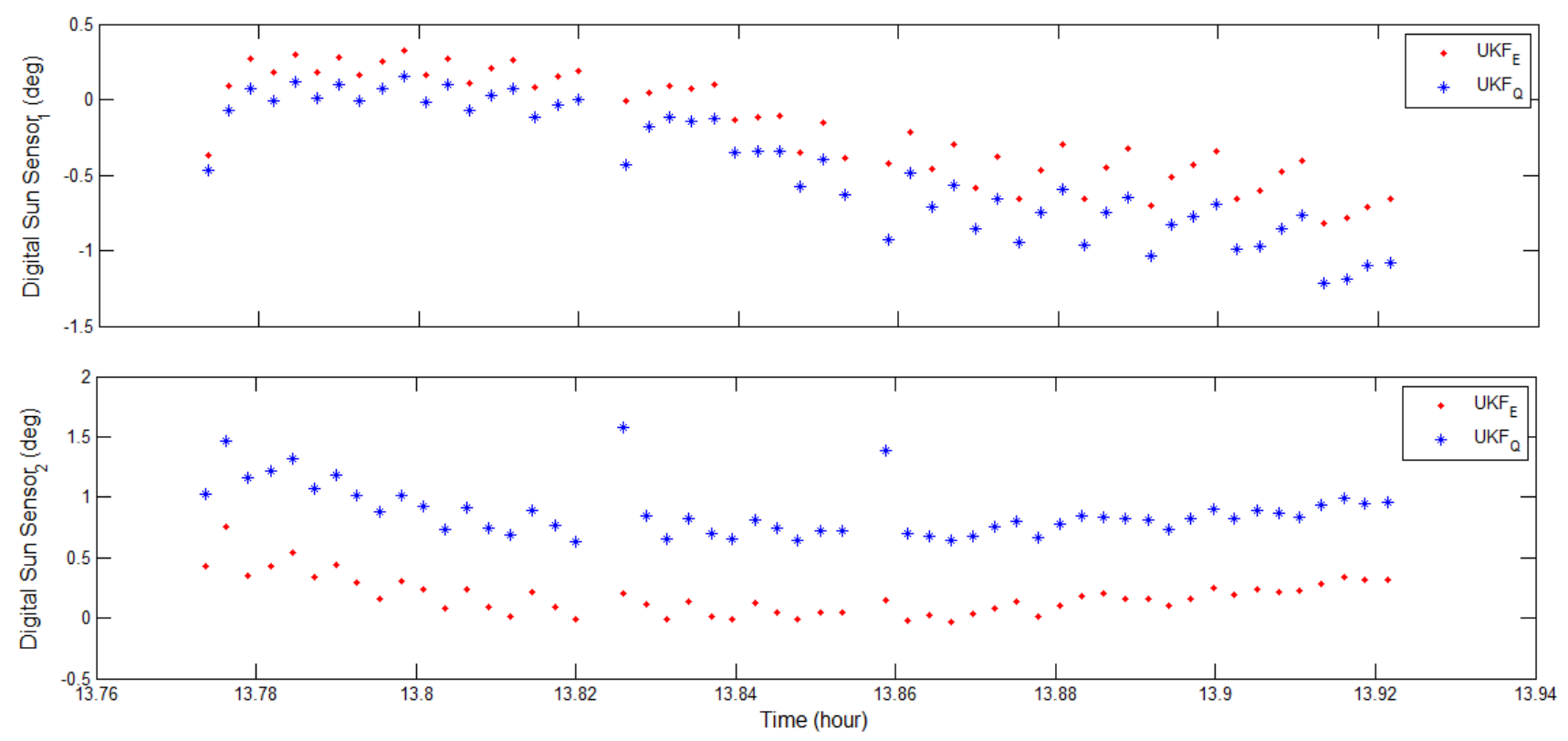

Figure 6. Residuals of Sun Sensors

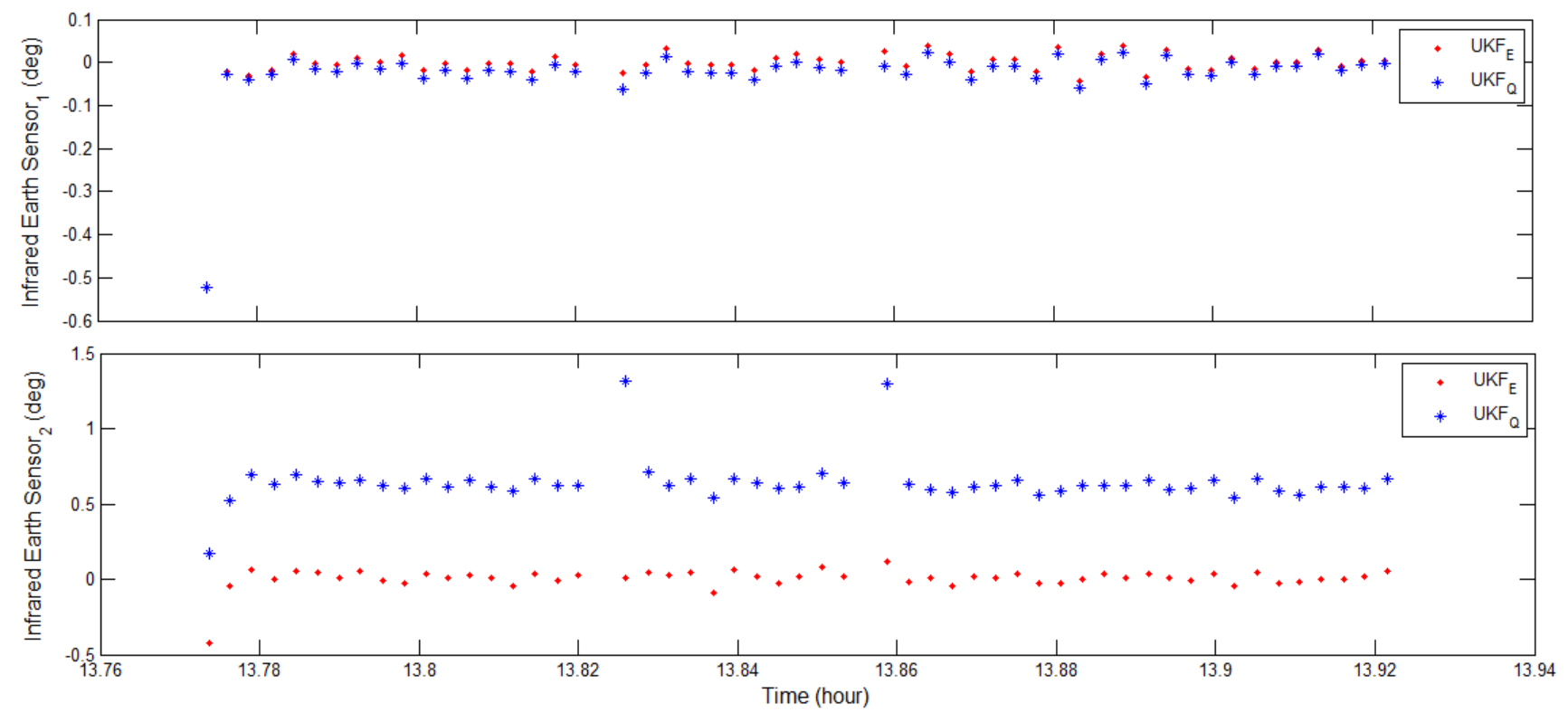

Figure 7. Residuals of Earth Sensors

\section{Final Comments}

The main objective of this study was to estimate the attitude of a CBERS-2 like satellite, using real data provided by attitude sensors besides using an approach to the nonlinear Kalman Filter, called Unscented Kalman Filter UKF [6]. This new filter is utilized considering two different parameterizations: the Euler angles $\mathrm{UKF}_{\mathrm{E}}$ and the quaternions $\mathrm{UKF}_{\mathrm{Q}}$. The results obtained were compared in order to analyze the advantages and disadvantages of each parameter, besides the performance of the results obtained by each filter. 
Although quaternions need 4 components with one redundancy when used in attitude estimation problems via unscented Kalman filter such parameterization makes the dynamical modeling linear. Therefore the overhead due to the unscented transformation to generate the sigma-points appears only in the measurement update cycle of the filter, where the measurement model is still nonlinear. This in principle translates to a computational saving because a numerical integration of $(2 n+1)$ sets of differential equations for the dynamics are avoided ( $n$ being the number of filter states), which is mandatory when using Euler angles. On the other hand, the number of states to be estimated increases and so the covariance as well. In a rough test to check CPU time, both the $\mathrm{UKF}_{\mathrm{E}}$ and $\mathrm{UKF}_{\mathrm{Q}}$ were run 100 times each, on the same $\mathrm{PC}$ in MatLab language. The $\mathrm{UKF}_{\mathrm{E}}$ took 14.26s of CPU time against $12.74 \mathrm{~s}$ of $\mathrm{UKF}_{\mathrm{Q}}$. Although $\mathrm{UKF}_{\mathrm{E}}$ was slower as expected the saving was not so significant to be conclusive.

Additionally it should be pointed out that the performance of the $\mathrm{UKF}_{\mathrm{Q}}$ depicts clearly that this filter parameters are still not well tuned. Some other considerations are in order such as quaternion normalization, quaternion covariance matrix singularity, numerical errors, and insufficient sampling rate to allow the hypothesis of constant angular velocity between samples. A future work in progress is considering the formulation of quaternion increments that might be another potential algorithm for utilization in the attitude determination problem.

\section{References}

[1] Lopes, R.V.F.; Kuga, H.K.; Silva, A.R., "CBERS 2 - Effects of Attitude Data in Image Quality.” São José dos Campos, INPE, Internal Report CBERS 2, C-ITRP, 2005.

[2] Crassidis, J. L., Markley, F. L., Cheng, Y., "Survey of Nonlinear Attitude Estimation Methods," Journal of Guidance, Control, and Dynamics, Vol. 30, No. 1, January-February, 2007.

[3] Crassidis, J. L., Markley, F. L., "Unscented Filtering for Spacecraft Attitude Estimation," Journal of Guidance, Control, and Dynamics, Vol. 26, No. 4, pp. 536-542, July-August, 2003.

[4] Fuming, H.; Kuga, H. K., "CBERS Simulator Mathematical Models." CBTT Project, CBTT /2000 /MM /001. INPE, São José dos Campos, 1999.

[5] Lefferts, E. J.; Markey, F. L.; Shuster, M. D., "Kalman Filtering for Spacecraft Attitude Estimation.” Journal of Guidance, Control and Dynamics, Vol. 5, No. 5, pp. 417-429, 1982.

[6] Julier, S. J.; Uhlman, J. K., "A new extension of the Kalman filter to non linear systems." Proceedings of the International symposium on aerospace/defense sensing, simulation and controls, Orlando, FL, 1997.

[7] Shuster, M. D., "A Survey of Attitude Representations," Journal of Astronautical Sciences, Vol. 41, No. 4, pp. 439-517, 1993.

[8] Wertz, J. R., "Spacecraft Attitude Determination and Control," D. Reidel, Dordrecht, Holland, 1978.

[9] Pisacane, V. L.; Moore, R. C., "Fundamentals of Space Systems." Oxford University Press, NY, 1994.

[10] Lopes, R.V.F.; Kuga, H. K., "CBERS-2: On Ground Attitude Determination from Telemetry

Data." São José dos Campos, INPE, Internal Report C-ITRP, 2005. 